\title{
Stillstand und Bewegung
}

\author{
Beyond Still/Moving. Fotografie und Film im Kunstdiskurs
}

\author{
»Es ist zwischen den Bildern, wo sich mehr und mehr die \\ Übergänge, die Kontaminationen von Wesen und Systemen \\ vollziehen: sie sind zuweilen ganz deutlich, zuweilen \\ schwer zu beschreiben und vor allem zu benennen. $\ll^{1}$
}

Raymond Bellour

Die bisher besprochenen Werkbeispiele haben gezeigt, dass in der zeitbasierten Medienkunst zeitliche Manipulationen des Bildes die traditionelle Scheidung in filmisches beziehungsweise videografisches Bewegtbild einerseits und malerisches, zeichnerisches oder fotografisches Standbild andererseits obsolet werden lassen. Dennoch hat sich in der entsprechenden Literatur der letzten drei Dekaden die Dichotomie von Stillstand und Bewegung als Begriffsfeld, in dem die hybriden Bildformen anzusiedeln sind, etabliert. In den folgenden Absätzen soll daher versucht werden, die theoretischen und historischen Hintergründe der Diskussion darzustellen, die unter den Stichworten Stillstand und Bewegung Eingang in die film- und kunsttheoretischen Diskurse gefunden hat und in der häufig eine Parallelisierung mit den medialen und zeitlichen Eigenschaften von Film und Fotografie stattfindet. ${ }^{2}$ Es geht hierbei um die Gründe dieser methodischen Gegenüberstellung und die Folgen für die kunsthistorische Arbeit an Werken, deren Intermedialität die Trennschärfe medienspezifischer Begrifflichkeiten aufweicht. Dass dabei eine Vermischung der repräsentativen Eigenschaften eines Bildes mit seinem materiellen Träger und der technischen Entstehungsweise entsteht, soll kritisch untersucht werden.

Für die Frage nach dem Wechselverhältnis von Zeitlichkeit und Geschichtlichkeit im bewegten Bild ist der Diskurs um die Dichotomie Stillstand und

1 Raymond Bellour, »Die Doppel-Helix«, in: Elisabeth von Samsonow, Éric Alliez (Hg.), Telenoia. Kritik der virtuellen Bilder, Wien 1999, S. 79-119, S. 8 o.

2 Zu einer ähnlichen Feststellung kommt Ingrid Hölzl: »A survey of recent literature indicates a need for a paradigm shift in the study of photographic images. Most of the literature does not call into question the opposition still/moving, but investigates instead the relation of still and moving images reduced to their dominant forms, photography and film.« (Ingrid Hölzl, »Editorial. Photography and Movement«, in: History of Photography 35/1, 2011, S. 2-5, S. 2.) 
Bewegung von großer Bedeutung, weil sich zwischen den beiden Extremen des Bildes zahlreiche Zustände des Übergangs, des Dazwischen manifestieren. Deren zeitliche Komplexität impliziert, dass die verwendeten Medien ebenfalls in zeitlicher Hinsicht mehrdimensional gedacht werden müssen: Die Historizität, die den Bildern qua ihrer Verweisung auf ihren Entstehungszusammenhang, auf Bildinhalte, vergangene Ereignisse oder Objekte eigen ist, trifft auf ihre Funktion innerhalb von Bildarchiven, die Dimension von Erinnerung, die Wertschätzung als Dokument. Schließlich ist zu fragen, ob die Bilder, die mit den Kategorien des Statischen und des Bewegten spielen, die Vergegenwärtigung von Vergangenem unterschiedlich leisten und unterstützen und wie sich dies in der Ästhetik der zeitbasierten Werke zeigt. Diese Komplexität in bildlicher, medialer und terminologischer Hinsicht hier aufzuzeigen erweist sich als wichtig für die Betrachtung der Werkbeispiele im weiteren Verlauf der Untersuchung, in dem das Augenmerk auf die Pose, das Found Footage und den filmischen Raum als Phänomene der Gegenwartskunst gelegt wird.

Zunächst sollen zwei Arbeiten näher betrachtet werden, denn nur die konkrete Anschauung erlaubt, die Terminologie und Struktur von hybriden Bildphänomenen überzeugend abzuleiten. Ein Film von Hollis Frampton, einem der wichtigsten Filmheoretiker und Experimentalfilmer der New Yorker Avantgarde der 196oer und 1970er Jahre, in dem er sich auf poetische Weise mit der Verschmelzung und Trennung von Fotografie und Film beschäftigt, steht einem Werk des Belgiers David Claerbout gegenüber, der mit digitaler Nachbearbeitung und Projektion stets verschiedene Zeitebenen des Bildes zueinander in Beziehung setzt.

In seinem Film (nostalgia) (Abb. 4) von 1971 wirft Hollis Frampton einen nostalgischen Blick auf die Fotografie und ihr Verhältnis zum Film, indem er in zwölf (beziehungsweise dreizehn) Bildern auf seine Karriere als Fotograf zurückblickt - von »the first photograph I ever made with the direct intention of making art « bis zu der Feststellung »I think I shall never dare to make another photograph again $\ll^{3}$ Dreizehn Schwarz-Weiß-Fotografien erscheinen nacheinander im Bild, verfärben und verformen sich langsam und gehen dann in Flammen auf, nachdem sie auf eine spiralförmige heiße Herdplatte gelegt wurden. Ein Voiceover, das von Michael Snow gesprochen ist, beschreibt die Bilder und erläutert deren Entstehungshintergründe. Es handelt sich zum

3 Diese und die folgenden sind, wenn nicht anders ausgewiesen, Zitate aus der Transkription des Voiceovers nach Hollis Frampton, »(nostalgia): Voice-over Narration for a Film of That Name«, in: ders., On the Camera Arts and Consecutive Matters. The Writings of Hollis Frampton, hg. v. Bruce Jenkins, Cambridge, MA 2009, S. 203-209. 

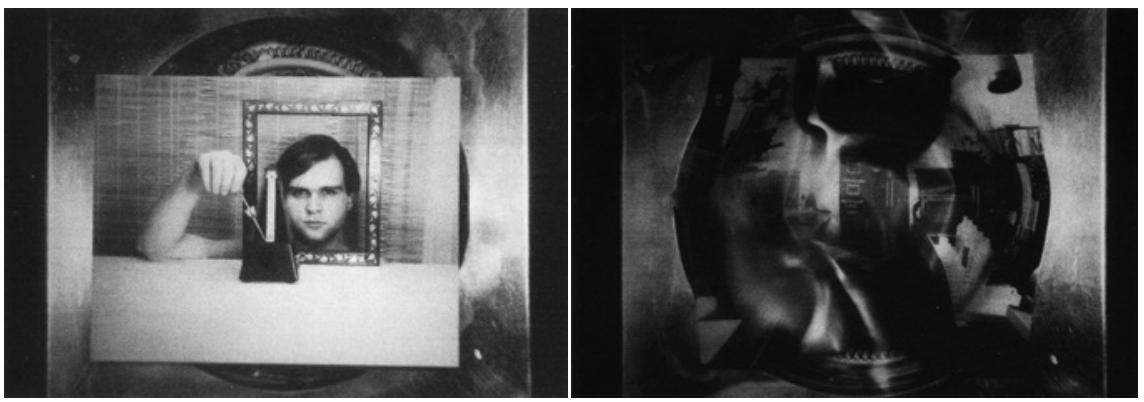

Abb. 4

Hollis Frampton, (nostalgia), 1971

Teil um Künstlerporträts (Carl Andre, Frampton selbst, Frank Stella, James Rosenquist, Michael Snow, Larry Poons), zum Teil um architektonische Schnappschüsse oder um urbane Szenen. Bei manchen hebt Frampton Details der bildlichen Komposition hervor, beschreibt einzelne Elemente und ihr visuelles Zusammenwirken, bei manchen teilt er den genauen Zeitpunkt und die Gelegenheit der Aufnahme mit oder die Gefühle, die er der Fotografie entgegenbringt (»I despised this photograph for several years«). Irritierend für den Betrachter / die Betrachterin ist vor allem die Asynchronität von Bild und Ton: Zur ersten Beschreibung eines Bildes - des Künstlers Carl Andre, wie er durch einen Bilderrahmen guckt und das Pendel eines Metronoms festhält sieht man nicht die beschriebenen Fotografie, sondern die Polaroidaufnahme einer fotografischen Dunkelkammer, welche unkommentiert bleibt (und in die Ankündigung »these are recollections of a dozen still photographs I made several years ago « nicht einbezogen wird). Das Bild von Andre erscheint in dem Moment, als die Beschreibung der darauffolgenden Aufnahme beginnt, und die Verschiebung von Bild und Text setzt sich bis zum Ende des Films fort. Dieser einfache und doch raffinierte Aufbau weist die Betrachter/innen auf die verschiedenen zeitlichen Dimensionen des fotografischen und filmischen Bildes hin, die hier ineinander verschachtelt werden: Die Fotografie, die in ihrem momenthaften Einfrieren des Zeitpunkts der Aufnahme stets in die Vergangenheit verweist - zu gleichen Teilen auf die fotografierte Realität wie auf den Moment der Bildentstehung -, wird vom Feuer unwiederbringlich zerstört; diesen Prozess können wir auf Film verfolgen, die Vergangenheit löst sich auf zugunsten der Gegenwart der Filmbetrachtung, wo wir der irreversiblen Zerstörung des Fotos beizuwohnen meinen. Das Voiceover jedoch verweist schon in die Zukunft, es beschreibt nie das aktuelle, sondern immer das noch kommende Bild. Erscheint diese Fotografie dann, müssen die Betrachter/innen auf ihre Erinnerung der vormals zukünftigen Beschreibung zurückgreifen und diese mit dem sich bereits wieder auflösenden Bild vergleichen. Dabei 
ertönt schon der Kommentar zum noch nicht sichtbaren Bild, der unweigerlich die Imagination anregt, es sich vorzustellen, und so fort. Ohne hier auf die mannigfaltigen Details der einzelnen Fotografien und ihrer Beschreibungen einzugehen, sind dem Aufbau der Arbeit einige wichtige Annahmen über die Konnotation der verschiedenen Medien Fotografie, Film und Sprache zu entnehmen. Es ist eine nostalgische Meditation über die Möglichkeiten der Fotografie, den herausgehobenen ebenso wie den beiläufigen Augenblick festzuhalten, was sie jedoch strukturell immer mit der Vergangenheit, diesem speziellen Augenblick der Aufnahme verbindet. Der Film indes zeigt die Fotografie als Repräsentation dieses Moments und verschmilzt für einen Augenblick mit der statischen Darstellung, bis die Zerstörung als Prozess dokumentiert wird.

Frampton sieht das strittige Verhältnis von Fotografie und Film als eine leidige Diskussion und vergleicht sie in seinem filmtheoretischen Essay »For a Metahistory of Film « mit der Huhn-Ei-Frage: Für ihn ist zwar die Fotografie vorher dagewesen und beschleunigt der Film nur die fotografischen Bilder in die Bewegung, umgekehrt fasst er den Begriff von Film jedoch so weit - er spricht von einem unendlichen Kino, dem »infinite cinema «, ${ }^{4}$ das alle potenziellen Bilder der Welt umfasst -, dass die Fotografie wiederum darin aufgeht. Für Frampton ist das Kino beziehungsweise der Film (der englische Ausdruck cinema kann bekanntlich sowohl Film als auch Kino bedeuten) das letzte Relikt des Zeitalters der Maschinen, also der Apparate, die nach physikalischen Gesetzen funktionieren und aus voneinander unterscheidbaren Teilen bestehen: »Cinema is the Last Machine. It is probably the last art that will reach the mind through the senses. $\aleph^{5} \mathrm{Er}$ hat einen essentialistischen wie auch einen historischen Blick auf die Kunst des Kinos, dessen Ende er nicht durch das Video, sondern bereits früher, durch die Entwicklung des Radars eingeläutet sieht. ${ }^{6}$ Das Radar als elektromagnetische Technologie, deren Signale nicht wie die optischen oder akustischen Signale durch die menschlichen Sinne aufgenommen werden können, ist für ihn in prädigitaler Zeit offensichtlich diejenige Technik, die im stärksten Gegensatz zum Film und seiner Sinnlichkeit, die er mit der Fotografie teilt, steht.

4 Hollis Frampton, »For a Metahistory of Film. Commonplace Notes and Hypotheses«, in: ders., On the Camera Arts and Consecutive Matters, S. 131-139, S. 134. Vgl. auch Mark B. Hansen, »Digital Technics Beyond the `Last Machine «. Thinking Digital Media with Hollis Frampton«, in: Eivind Røssaak (Hg.), Between Stillness and Motion. Film, Photography, Algorithms, Amsterdam 2011, S. 45-72.

5 Frampton, »For a Metahistory of Film«, S. 136.

6 »I prefer radar, which replaced the mechanical reconnaissance aircraft with a static, anonymous black box.« (Ebd.) 
Eine Generation später nutzt David Claerbout gerade die digitale Technik, um Fotografie und Film im digitalen Video erneut miteinander zu konfrontieren. In einer seiner frühen Arbeiten, Kindergarten Antonio Sant' Elia, 1932, von 1998, verwendet er eine historische Schwarz-Weiß-Fotografie - der Titel suggeriert als Entstehungszeit das Jahr 1932 - von spielenden Kindern in einheitlichen weißen Kitteln, die von einem erhöhten Standpunkt aus in einem Garten aufgenommen wurde (Abb. 5). Der Garten ist durch eine Mauer begrenzt, ein gestutzter Rasenstreifen trennt zwei akkurat gepflasterte Flächen, in denen zwei junge Bäume stehen. ${ }^{7}$ Projiziert als Videostill, nimmt der Betrachter oder die Betrachterin zunächst den Gegensatz zwischen der formalistischen Strenge des Gartens und der Ungeordnetheit der sich offenbar im bewegten Spiel befindenden Kinder wahr. Es sind jedoch die Blätter der Bäume, die sich tatsächlich fast unmerklich bewegen, die nachträglich animiert wurden und der Stillstellung der Fotografie ein sanftes Flimmern entgegensetzen. Claerbout spielt hier eventuell auch auf einen Topos aus der Frühzeit des Kinos an, als die Möglichkeiten der Kinematografie, die Bewegung und das Zittern der Blätter im Wind darzustellen, als Besonderheit des Films hervorgehoben wird. ${ }^{8}$ Auch in seinem weiteren Werk verfolgt Claerbout immer wieder das Ziel, die verschiedenen Zeitebenen von Fotografie und bewegtem Bild (er arbeitet stets mit Video und digitaler Projektion, nicht mit analogem Film) miteinander zu kombinieren und die Spannung, die dabei entsteht, ins projizierte Bild zu übertragen: »I look at moving images with the body of a still camera, and I look at photography with the contingency of movement, all for the sake of finding a moment that will evolve on itself, while being suspended in time. $\ll^{9}$ Ebenso wie Frampton und die anderen hier vorgestellten Künstler/innen interessiert er sich für das Zusammenspiel von Gegenwärtigkeit und Vergangenheit im Bild. Gerade in der Latenz des projizierten Videobilds, durch die er auch auf die räumliche Mobilität und zeitliche Flüchtigkeit der Projektion anspielt,

7 Es handelt sich um den Garten des gleichnamigen Kindergartens in Como, der von dem Architekten Giuseppe Terragni gestaltet wurde (vgl. David Claerbout, Uncertain Eye, Ausstellungskatalog Pinakothek der Moderne, München, München 2010/2011, S. 29). Der rationalistische Architekt Terragni, der den italienischen Faschisten nahestand, erhielt $193^{2}$ den Auftrag für den Kindergarten, das Gebäude wurde jedoch erst $1935^{-1937}$ errichtet. Die Datierung der Fotografie ist daher womöglich etwas später anzusetzen.

8 Henri de Parville, »Le Cinématographe«, in: Les Annales politiques et littéraires, 26.4.1896, S. 269-270, S. 270: »Les photographies animées sont de petites merveilles. On distingue tous les détails, les tourbillons de fumée qui s'élèvent, les vagues de la mer qui viennent se briser sur la plage, le frémissement des fouilles sous l'action de la brise, etc.«

9 Christine van Assche, David Claerbout, »Interview«, in: Christine van Assche (Hg.), David Claerbout. The Shape of Time, Ausstellungskatalog Centre Georges Pompidou, Paris 2007/2008, Zürich 2008, S. 9-15, S. 13. 


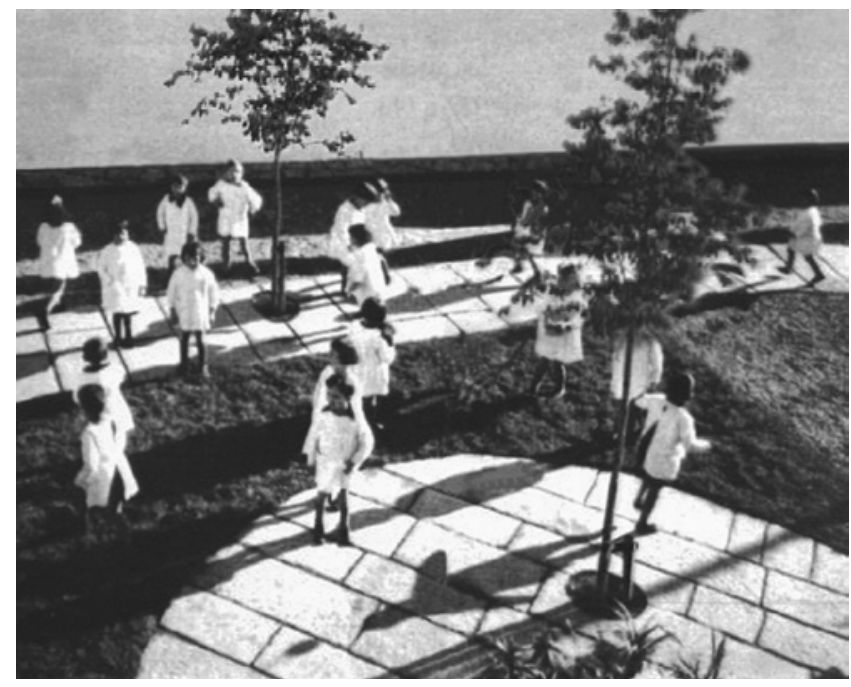

Abb. 5 David Claerbout, Kindergarten Antonio Sant' Elia, 1932, 1998

zeigt sich das Bewegungspotenzial des Bildes als »invertierte Energie«, wie folgendes Zitat verdeutlicht:

But if I merely add movement to the still, the picture will collapse under the pressure of it. Instead I prefer to think of alterations as >caresses< on the surface, in order to stay in contact with both the picture's past and present. The videoprojected still is full of potential for movement, and it is precisely this snot happening $<$ which is an inverted energy, not a forward moving energy. ${ }^{10}$

Es ist primär auf die gemeinsame technische Grundlage der Projektion auf lichtempfindliches Material zurückgeführt worden, dass gerade das Verhältnis von Fotografie und Film so sehr im Fokus des wissenschaftlichen und populären Interesses insbesondere der letzten zwei Dekaden, aber auch in der frühen Filmtheorie steht. Dass »der Film im wesentlichen eine Erweiterung der Fotografie «11 sei, stellte Kracauer an den Beginn seiner Filmtheorie, und so wahr diese Aussage auf der einen Seite ist, so falsch ist sie auf der anderen Seite: Zwar beruht der fotografische Film (im Gegensatz zum animierten Film, der aus der Zeichnung entwickelt wird) auf der genannten gemeinsamen technischen Grundlage, jedoch unterscheidet er sich zugleich in seiner Handhabung, Ästhetik, Theoriebildung, Verbreitung und nicht zuletzt in seinem

$10 \quad$ Ebd., S. 9.

11 Kracauer, Theorie des Films, S. 11. 
Verhältnis zur Zeit stark von der Fotografie. Die notwendigen Differenzierungen dominieren denn auch die Diskussionen, schreibt auch David Green:

Whatever arguments may be mustered on behalf of cinema's debts to literature and theatre, the technological bases of film have guaranteed photography a primary role in any account of its early development and perhaps continue to inflect an understanding of film as being - first and foremost - a pre-eminently visual medium. But the fact that photography and film have always been seen as closely intertwined has also proved to be the spur to differentiate between them. That this process of the differentiation of photography and film has revolved around a polarisation between the still and the moving image, and the different temporalities associated with each, should come as no surprise. ${ }^{12}$

Die verschiedenen Zeitlichkeiten, die den Medien Fotografie und Film zugeschrieben werden, beinhalten zunächst die prominente Verknüpfung der Fotografie mit dem Aspekt der Stillstellung des bewegten Objekts im Bild, indem sie einen Schnitt durch die Zeit fixiert, dann auch ihren Bezug zur Vergangenheit, die bereits oben erörterte Indexikalität, die als zeitliche Verbindung zur Realität der Vergangenheit gedeutet wird. Es ist nicht die Mimetik, die Möglichkeit des Wiedererkennens von etwas Vergangenem im gegenwärtigen Bild, sondern die verweisende und bezeugende Rolle der Fotografie, die dabei als spezifisch fotografisch hervorgehoben wird. Philippe Dubois geht noch weiter, indem er die Pierce'sche Indexikalität untrennbar mit dem Akt des Fotografierens, also dem Drücken des Auslösers, verknüpft: Dieser fungiere als performativer »Bild-Akt «, der den Augenblick dem Kontinuum der Zeit entreißt, als Schnitt in die Zeit, dessen »automatische Genese « (Bazin) »das Subjekt selbst voll und ganz in die Erfahrung, in das Erlebnis des fotografischen Prozesses einbezieht $\ll::^{13}$

Es gehört zu den entscheidenden Konsequenzen dieser Logik des Index, daß sie das fotografische Bild radikal als etwas setzt, was nur in Verbindung mit dem Akt seiner Hervorbringung denkbar ist, mag es sich dabei um den Akt der Rezeption, der Produktion oder um den Referenten des Bildes handeln. Als eine Art absoluter, mit seiner referentiellen Situation untrennbar verbundener Bild-Akt, erweist sich die Fotografie als von grundlegend pragmatischer Natur: ihr Sinn liegt zunächst in ihrer Referenz. ${ }^{14}$

12 David Green, »Marking Time. Photography, Film and Temporalities of the Image«, in: ders., Lowry, Stillness and Time, S. 9-22, S. 10.

13 Philippe Dubois, Der fotografische Akt. Versuch über ein theoretisches Dispositiv, Amsterdam 1998 (1983).

14 Ebd. 
Dem gegenüber steht die Assoziation des Films mit der Bewegung, die nach Christian Metz den Hauptunterschied zwischen Film und Fotografie ausmacht. Wo der Film eine »zeitliche Größe« (»temporal size«), Bewegung und Pluralität (»movement and plurality«) zeige, sei die Fotografie zeitlos (»timelessness«) und durch Bewegungslosigkeit und Stille (»immobility and silence«) gekennzeichnet. ${ }^{15}$ Die Bewegung ist nach Metz maßgeblich dafür verantwortlich, dass die Zuschauer/innen das Leinwandgeschehen als lebendig und somit >reak wahrnehmen. Ihr ist der besondere Realitätseindruck des Kinos zu verdanken, welcher wiederum die Filmwahrnehmung an die Gegenwart bindet:

[E]s ist die Bewegung (einer der größten Unterschiede, zweifellos der größte zwischen dem Kino und der Photographie), die Bewegung erzeugt einen starken Realitätseindruck. Diese Antwort ist selbstverständlich schon oft gegeben worden, aber vielleicht nicht vollständig. $>$ Die Verbindung zwischen der Realität der Bewegung und der Erscheinung der Formen erzeugt das Gefühl konkreten Lebens und den Eindruck einer objektiven Realität<, ${ }^{16}$

zitiert Metz Edgar Morin in Le cinema ou l'homme imaginaire ${ }^{17}$. 2007 hat Tom Gunning Metz' Primat der Bewegung über der Indexikalität wieder aufgegriffen, um es für die Analyse verschiedener theoretischer und ästhetischer Aspekte der Kinotheorie fruchtbar zu machen. ${ }^{18}$ Das Augenmerk auf die Bewegung zu richten ermögliche es - was Gunning nur vorschlägt, aber nicht ausführt -, die Betrachtungssituation, Filmstile und die Einbindung neuer Medien neu zu überdenken; ebenso könne die bisher in der Filmtheorie marginalisierte Animation gleichberechtigt einbezogen werden. Prozesse der Immersion, der Affizierung und der Fiktionalität, so kann hinzugefügt werden, hängen maßgeblich von der Bewegung des Bildes und ihrer Wahrnehmung ab. ${ }^{19}$

\section{Fotografie und Film bei Roland Barthes}

Bewegung und Stillstellung in Fotografie und Film sind in den $1980 e r$ Jahren vor allem in der französischen Semiologie diskutiert worden. Neben Metz waren

15 Vgl. Christian Metz, »Photography and Fetish«, in: October 34, Herbst 1985, S. 81-9o, S. $81-83$.

16 Metz, »Zum Realitätseindruck im Kino«, S. 25.

17 Edgar Morin, Le cinema ou l'homme imaginaire. Essai d'anthropologie, Paris 1956, S. 123.

18 Vgl. Tom Gunning, »Moving Away from the Index. Cinema and the Impression of Reality«, in: differences $18 / 1,2007$, S. 29-52, S. 38 .

19 Vgl. hierzu u. a. Marie-Luise Angerer, »Affektiv. Zur produktiven Differenz des Intervalls« sowie »Zur Technizität des Affektverkehrs im Bewegtbild. Verschränkungen von Mensch und Medium bei The Clock«, in: Pirkko Rathgeber, Nina Steinmüller (Hg.), BildBewegungen/ImageMovements, Paderborn 2013, S. 55-74 und 232-254. 
und sind die Schriften von Barthes bis heute besonders einflussreich. Dieser hat in seinem fototheoretischen Essay Die helle Kammer zahlreiche Eigenschaften der Fotografie beschrieben, die schon von früheren Theoretikern mit dem Medium verknüpft wurden: die direkte, indexikalische Verbindung zur Natur über das Licht (»Nabelschnur«), die zeitliche Dimension der Vergangenheit im Bild, die Zeugenschaft, die Möglichkeit, über Vergrößerung und das Detail die Wirklichkeit besser zur erkennen, als es mit bloßem Auge der Fall ist. ${ }^{20}$ Der Bezug zu Kino und Film ist in Die helle Kammer marginal, aber umso aufschlussreicher, als Barthes keineswegs seiner der Fotografie zugeschriebenen Verbindung zum Tod dichotomisch die Lebendigkeit des Films gegenüberstellt. Vielmehr beschreibt er einige Unterschiede: die Rezeption, die von Zerstreuung anstatt von Kontemplation gekennzeichnet ist, ${ }^{21}$ die räumliche Öffnung des bewegten Bildes ins hors-champ, das heißt das Vorhandensein eines Off außerhalb des Rahmens der Leinwand, ${ }^{22}$ schließlich die Flüchtigkeit des fotografischen Referenten, den zu fassen dem Zuschauer schwerfällt.. ${ }^{23}$ Interessanter ist in dieser Hinsicht Barthes' Diskussion einiger Eisenstein-Filme in dem Text »Der dritte Sinn «, wo er versucht, etwas Ähnliches wie das punctum der Fotografie an bestimmten Einstellungen des Films festzumachen. Was Barthes als den sstumpfen Sinn des Films $<$ beschreibt, ist neben der informativen und der symbolischen Ebene eine dritte, poetische Ebene des bildlichen Inhalts, die letztlich das spezifisch Filmische (und damit für ihn genuin Bildliche) ausmacht, denn hier versagt die Sprache: »[D]er stumpfe Sinn ist ein Signifikant ohne Signifikat; daher die Schwierigkeit, ihn zu benennen « ${ }^{24}$ Barthes führt weiter aus: »Das Filmische ist dasjenige im Film, das sich nicht beschreiben läßt, die Darstellung, die sich nicht darstellen läßt. Das Filmische beginnt erst dort, wo die Sprache

$20 \quad$ Als close reading empfiehlt sich das erste Kapitel von Florian Arndtz, Philosophie der Fotografie, Paderborn 2013.

21 »Füge ich auch dem Bild etwas hinzu? Ich glaube nicht, dafür bleibt mir keine Zeit: vor der Leinwand kann ich mir nicht die Freiheit nehmen, die Augen zu schließen, weil ich sonst, wenn ich sie wieder öffne, nicht mehr dasselbe Bild vorfände; ich bin zu ständiger Gefräßigkeit gezwungen« (Roland Barthes, Die helle Kammer. Bemerkungen zur Photographie, Frankfurt am Main 1989, S. 65).

22 Ebd., S. 66. Bildern, die ein punctum besitzen, gesteht Barthes ein solches »blindes Feld« zu, eine zweite Bedeutungsebene, die jedoch nicht räumlich in Bezug zum Bildrahmen zu verorten ist.

23 Ebd., S. 100.

24 Roland Barthes, »Der dritte Sinn. Forschungsnotizen über einige Fotogramme S. M. Eisensteins « (1970), in: ders., Der entgegenkommende und der stumpfe Sinn. Kritische Essays III, Frankfurt am Main 2005, S. 47-66, S. 6o. 
und die gegliederte Metasprache aussetzen. ${ }^{25}$ Dieser >stumpfe Sinn $<$ ist also im Gegensatz zum >entgegenkommenden Sinn<, der als Bedeutungsebene des Films benenn- und beschreibbar ist, reine Signifikanz, eine Stumpfheit, an der der Blick nicht abgleitet, sondern hängenbleibt. Paradoxerweise - wie Georges Didi-Huberman angesichts der Tatsache bemerkt, dass Barthes sich auf Eisensteins Montagetheorie bezieht ${ }^{26}$ - verortet Barthes den sstumpfen Sinn< im filmischen Einzelbild, das für ihn die einzige Möglichkeit bietet, über das Filmische zu sprechen. Seiner Vorliebe für die Fotografie entsprechend ist es gerade nicht die Bewegung, die Barthes als das spezifisch Filmische isoliert, sondern etwas, das sich nur im Fotogramm zeige (in seiner französischen Bedeutung als Einzelbild aus einem Filmstreifen, nicht als Ergebnis kameraloser Fotografie). Einerseits schreibt Barthes die Fixierung aufs Fotogramm seinem eigenen Unvermögen und seiner $»$ filmischen Ungebildetheit ${ }^{27} \mathrm{zu}$, andererseits jedoch meint er in der einmaligen Verbindung des Diegetischen mit dem Bildlichen, die im Fotogramm aufscheint, das eigentlich Filmische zu entdecken. Die Bewegung sei »nur das Gerüst einer permutativen Entfaltung ${ }^{28}{ }^{28}$ die auf der von Eisenstein selbst so genannten vertikalen Lektüre des Bildes beruht. ${ }^{29}$ Die Filmzeit, also das kontinuierliche Fortlaufen des Films, sieht Barthes als lästigen Zwang an, der einer adäquaten Lektüre der filmischen Fragmente im Weg steht ${ }^{30}$ - ein weiteres Indiz dafür, dass Barthes auch in seinen wenigen filmbezogenen Schriften dem statischen Einzelbild den Vorzug gibt.

\section{Freeze Frame}

Bezugnehmend auf Barthes und dessen Charakterisierung der Fotografie haben Jacques Aumont und Raymond Bellour als Erste die Frage nach der Stillstellung des Bildes systematisch erörtert. Unter dem Einfluss der Verbreitung der Videotechnik, die nicht nur die Filmerfahrung und -analysemöglichkeiten radikal veränderte, sondern das Verhältnis zum Film und zum bewegten Bild

25 Ebd., S. 63.

26 Georges Didi-Huberman, »Klagebilder, beklagenswerte Bilder? «, in:Zeitschrift für Medienwissenschaft 1, 2009, S. 51-6o, S. 55 f.

27 Barthes, »Der dritte Sinn«, S. 64.

28 Ebd., S. 65 .

29 Die sogenannte Vertikalmontage beschreibt das zeitgleiche Zusammenwirken von Ton und Bild, wobei die Bewegung »als Kompositionsgesetz« beidem zugrunde liegt. Eisenstein orientiert sich am Modell der Partitur, die ebenfalls einerseits horizontal, andererseits vertikal gelesen werden muss. Vgl. Sergej M. Eisenstein, »Die Vertikalmontage«, in: ders., Jenseits der Einstellung. Schriften zur Filmtheorie, hg. v. Felix Lenz und Helmut H. Diedrichs, Frankfurt am Main 2006, S. 238-300.

Vgl. Barthes, »Der dritte Sinn«, S. 66. 
allgemein neu definierte, wurde das angehaltene Bild - »l'arrêt sur image, ou de l'image « zum Phänomen, an dem sich die Kollision verschiedener Medien im Bild selbst zeigte: »Ce n'est pas un hasard si le développement de l'arrêt sur image et de toutes les formes du photographique, qui envahit le cinéma au tournant des années 6o, a coïncidé avec les transformations qui se sont précisées depuis grâce au traitement de l'image électronique.«11 Bellour widmet sich im Speziellen der Frage nach Stadien der Stillstellung des Bildes im Film. ${ }^{32}$ Anhand verschiedener Beispiele richtet er dabei seine Aufmerksamkeit zunächst auf den arrêt sur l'image oder Freeze Frame, das Phänomen des angehaltenen Standbilds innerhalb eines Films, dann mit Roland Barthes' »drittem Sinn « auf das Fotogramm (das Einzelbild aus einem Filmstreifen), schließlich auf das Erscheinen von Fotografien im Spielfilm, eine weitere Art der Stillstellung innerhalb bewegter Bilder. Anhand dieser sehr unterschiedlichen Phänomene versucht Bellour, Aussagen über das Verhältnis von Fotografie und Film zu treffen: Es zeige sich, dass die Fotografie immer »fruchtbare « Augenblicke produziere und »always by the force of circumstance a >decisive instant torn from reality « $33^{33}$ sei. Der Film hingegen bestehe seinem Wesen nach eben nicht aus herausgehobenen Momenten, dennoch könnten wir solche wahrnehmen, denn es höben sich manche Bilder und Szenen heraus aus dem Fluss der Bilder. Diese neigten, so Bellour, entweder zum Fotografischen und somit zur Kraft, den Tod einzuschreiben, oder aber zeichneten sich durch eine bestimmte Abstraktheit und Irrealität aus:

[T] he instant that stills the film bears a relation to the film as a whole. It goes way beyond its purely material inscription, reverting the film back onto itself, capturing its singular drama, emphasizing the fact that it cannot be reduced to the overly natural time of illusion, inducing a time-space at the juncture of the visible and the invisible. ${ }^{34}$

Bellour hat untersucht, inwiefern sich dadurch die Rolle der Betrachter/innen verändert, und sie als >spectateurs pensifs $<$, als nachdenkliche Betrachter/ innen bezeichnet. Besonders funktioniere dies bei Fotofilmen wie beispielsweise dem berühmten La Jétée (1963) von Chris Marker:

Quelle différence, dès lors, dans ces films où la matière de la photo devient la fiction du cinéma? Tout simplement ceci: leur fixité relative adoucit >l'hystérie< du film. [...] La présence de la photo, diverse, diffuse, ambiguë, a ainsi comme

31 Raymond Bellour, »L'Entre-Images« (1990), in: ders., L'Entre-Images, S. 11-17, S. 13.

32 Raymond Bellour, »The Film Stilled«, in: Camera Obscura 24, 199o, S. 99-123.

33 Ebd., S. 108.

34 Ebd. 
effet de décoller (même de façon minimale) le spectateur de l'image [...]. Elle arrache le spectateur à cette force peu précise mais prégnante: la moyenne imaginaire du cinéma. ${ }^{35}$

Fotografie als Bestandteil des Films ermöglicht so paradoxerweise eine größere Reflexion über die Mechanismen des Kinos, als dieses es selbst zu leisten imstande ist. Der arrêt sur l'image als plötzliche Unterbrechung des gewohnten Fließens der Bilder lässt die Zuschauer/innen zurückprallen, die durch kontinuierliche Wiederholung eines einzelnen Filmstandbildes erreichte Bewegungslosigkeit unterscheidet sich radikal von dem Filmen unbewegter Situationen, denen immer noch die Zeitlichkeit und Bewegung der Realität anhaftet (vorausgesetzt, der Film wird in entsprechender Geschwindigkeit abgespielt). Diese Bilder sehen uns an, schreibt Bellour, und sie scheinen der Zeit zu widerstehen: »Elles ouvrent en fait un autre temps: un passé du passé. Un temps second et différent. Ainsi elles fixent un instant le temps du film; nous arrachant a son déroulement, elles nous situent par rapport à lui. « ${ }^{36}$ Bellour schreibt also dem arrêt sur l'image eine stark reflexive Funktion und Kapazität zu: Es ermögliche, die Wirkung der Integration unterschiedlicher Medien zu beobachten, wodurch sich die Wahrnehmung der Zeit und die Selbstwahrnehmung der Betrachtenden während des Filmschauens verändere.

\section{Geschwindigkeit}

Wurde zur Zeit der Filmavantgarde vorwiegend mit Geschwindigkeiten der Bilder durch Zeitlupen, Freeze Frames, Beschleunigung und Tricks der Belichtung experimentiert, eröffneten sich ab Mitte der 196oer Jahre durch die Verbreitung des Videos und ab den 199oer Jahren durch den Computer immer mehr Möglichkeiten, die Grenzen zwischen den klassischen Medien verschwimmen zu lassen. Für die französische Film- und Fototheorie konstatiert Philippe Dubois eine Zäsur um das Jahr 199o, von der an die in den 1970er und 1980er Jahren populäre und einen gegenseitigen Ausschluss voraussetzende Gegenüberstellung von Fotografie und Film, von Stillstellung und Bewegung, vertreten durch die populären Konzepte des punctum bei Barthes und des Bewegungs- und Zeit-Bildes bei Deleuze, nicht mehr galt. ${ }^{37}$ Die Verbreitung des

37 »D'un côté, Roland Barthes dans La Chambre claire imposait le concept de >punctum< en jouant la photographie contre le cinéma [...]. D'un autre côté, la philosophie bergsonodeleuzienne du cinéma imposait les concepts d'image-mouvement et d'image-temps, qui reposait encore entièrement sur l'idée que le film est un défilement régulier d'images reproduisant le mouvement apparent [...]. Tout se passait comme si l'un et l'autre, le 
Videos und schließlich des digitalen Bildes hätten in den folgenden Dekaden die alten Dichotomien obsolet werden lassen, denn damit einhergehend seien auch die zeitlichen Regimes verändert, ausgedehnt und aufgeweicht worden: »[L]es régimes temporels d'images se sont considérablement élasticisés, rendant de plus en plus obsolètes ou indiscernables les vieux partages. « ${ }^{38}$ Über die Gegenüberstellung von Fotografie und Film hinaus könne jetzt nur noch über Formen von Bildern gesprochen werden, die diese Aufteilung in das vorige Jahrhundert verwiesen, sie obsolet werden ließen. Unbewegtes, das sich bewegt (als Beispiel nennt er die Pose oder das Panorama), Bewegtes, das sich quasi nicht bewegt, und ständig befinde man sich dazwischen, »dans le va-etvient intermédiaires, dans l'espace-temps indécidable de ralentis-accélérés one ne fait même plus la différence - systématiques «.39

Diese neue Art Bilder sei mitnichten vollkommen neu: Historische Vorläufer, die sich mit der Geschwindigkeit von Bildern beschäftigt hätten, seien zum Beispiel Étienne-Jules Marey, Dziga Vertov, Jean Epstein oder Jean-Luc Godard. ${ }^{40}$ Die hier beschriebene zeitliche und mediale Entgrenzung und Hybridisierung von Bildern nahm jedoch ihren Anfang in den 196oer Jahren und lenkte in Reaktion auf das Primat der Medienspezifik des Modernismus das Augenmerk der Theoriebildung auf die Frage nach der Intermedialität der Kunst.

\section{Medienspezifik und Intermedialität}

Theorien der Intermedialität beschäftigen sich mit dem Verhältnis der Korrelation, Interaktion und Überschneidung von Medien. Der Begriff wird einerseits für die Synthese von Medien verwendet, also die Verschmelzung zu etwas Neuem, in dem die früheren Formen nicht mehr ohne Weiteres voneinander zu trennen sind - dies bezeichnet der Fluxuskünstler Dick Higgins in seinem frühen Aufsatz »Intermedia« als Intermedialität, die er von Mixed Media unterscheidet, also einer Art der Multimedialität, in der jedoch die ursprünglichen Formen wieder voneinander zu unterscheiden sind. ${ }^{41}$ Neben dieser Art synthetisierender Intermedialität nennt Jens Schröter noch drei

mobile et l'immobile, le fixe et le mouvant, ne pouvaient exister que dans un rapport d'exclusion réciproque.« (Philippe Dubois, »Entre cinéma et photographie: quelques variations de vitesse de l'image contemporaine «, in: Jerôme Game [Hg.], Images de corps / corps des images au cinéma, Lyon 2010, S. 91-108, S. 92.)

38 Ebd.

39 Ebd., S. 93 .

40 Ebd.

41 Vgl. Dick Higgins, »Intermedia« (1965), in: Leonardo 34/1, 2001, S. 49-54. 
andere Formen von Intermedialität: ${ }^{42}$ Transmedialität, also medienübergreifende Gemeinsamkeiten einzelner Darstellungsweisen; »transformative Intermedialität« im Sinne von »Re-Representation«, also der Umstand, »daß ein Medium ein anderes repräsentiert«; ${ }^{43}$ schließlich, was er als Gegenteil von Letzterem bezeichnet, eine ontologische Intermedialität. In ihr bestimmt sich ein Medium durch reflexive Bezugnahme auf andere Medien selbst. ${ }^{44}$ In Bezug auf das Verhältnis von Fotografie und Film, wie es in Werken der zeitgenössischen Kunst und deren Rezeption thematisiert wird, ist die zweite von Schröter diskutierte Art der Intermedialität relevant, nämlich in Form der Frage, inwiefern sich medienübergreifende Gemeinsamkeiten - auch als historische Phänomene - feststellen lassen. Diese beziehen sich nämlich nicht auf eine Vermischung von Techniken oder Künsten, sondern auf Aspekte, die den unterschiedlichen Medien eigen sind und dort jeweils spezifische Ausformungen erfahren. Jacques Aumont hat beispielsweise als Gemeinsamkeit von Malerei, Panorama, Fotografie und Kino eine am Beginn der Moderne, also an der Schwelle des 19. Jahrhunderts, neue Variabilität des Auges beziehungsweise eine Mobilisierung des Blicks beschrieben; ${ }^{45}$ er fasst sie zusammen als Dispositive des Sehens, in denen der Betrachter / die Betrachterin fixiert, das Auge jedoch beweglich ist. Ähnlich beschreibt Jonathan Crary in Techniques of the Observer (Techniken des Betrachters) die vergleichbaren Sehgewohnheiten von impressionistischer Malerei, Fotografie und Film mit Foucault als >Ordnung des Sichtbaren< oder in Bezug auf Martin Jay als skopisches Regime. ${ }^{46}$ In den folgenden Kapiteln werden die Figur der Pose, die Arbeit mit Found Footage und die Heterochronie filmischer Räume im Fokus stehen: Auch hier wird auf den Begriff der Intermedialität zurückzukommen sein, denn an allen drei Phänomenen kann man die Berührung und Überschneidung von Medien wie auch die medienübergreifenden, bildlich zu nennenden Modi der Darstellung beobachten. Die Pose, um ein Beispiel vorwegzunehmen, wird als Figuration von Bewegung in unterschiedlichen Medien erfahrbar und somit als vergleichendes Element der Video-, Fotografie- und Filmarbeiten

42 Jens Schröter, »Intermedialität. Facetten und Probleme eines aktuellen medienwissenschaftlichen Begriffs«, in: montage AV 7/2, 1998, S. 129-154.

43 Ebd., S. 144.

44 Ebd., S. $146 \mathrm{f}$.

45 Jacques Aumont, »L'oeil variable, ou la mobilisation du regard«, in: ders., L'Oeil Interminable. Cinéma et Peinture, Paris 1995, S. 37-72.

46 Jonathan Crary, Techniques of the Observer. On Vision and Modernity in the Nineteenth Century, Cambridge, MA 1990; Michel Foucault, Die Ordnung der Dinge. Eine Archäologie der Humanwissenschaften, 18. Auflage, Frankfurt am Main 2003; Martin Jay, »Scopic Regimes of Modernity«, in: Hal Foster (Hg.), Vision and Visuality, Seattle 1988, S. 3-28. 
von Coleman und Dean fruchtbar gemacht. Sie stellt nach Schröters Definition ein Phänomen dar, in dem die »formalen Ebenen getrennt von der medialen Basis und relativ autonom ihr gegenüber « 47 fungieren; aber eben nur relativ, denn um als Form am Körper sichtbar zu werden, muss die Pose sich im jeweiligen Medium aktualisieren, ${ }^{48}$ und nur so können auch die historischen Dimensionen der posierenden Darstellung, ihre fotohistorischen und -theoretischen Implikationen zutage treten.

In den zu Beginn kurz skizzierten künstlerischen Beispielen von Hollis Frampton und David Claerbout geht es ebenfalls nicht um die Möglichkeit des Films, die Fotografie zu vereinnahmen und sich ihre Darstellungsstrukturen anzueignen oder umgekehrt die filmische Bewegung komplett in eine Fotografie zu integrieren. Ganz im Gegenteil sind in beiden Arbeiten die Elemente des jeweiligen Mediums erkennbar. Die Fotografie hat in Framptons Film die Aufgabe, die Vergänglichkeit des fotografischen Moments zu symbolisieren und die Zerstörung des fotografischen Beweises zu dokumentieren. Dies ist wiederum (bis zu dessen Zerstörung) auf Film gespeichert und stets wieder aufführbar, womit sich die zeitliche und indexikalische Verknüpfung von Bild und Ereignis fortsetzt. Bei Claerbout haben die bewegten Blätter eine allegorische wie auch diskurshistorische Bedeutung, indem sie auf den Topos der bewegten Blätter in der Filmgeschichte anspielen. Beide Künstler verbinden verschiedene Medien, belassen jedoch ihre spezifische ästhetische, historische und diskursive Konnotation sichtbar.

\section{Terminologie}

Diese Frage nach einer Spezifik der Medien hat im Diskurs um Fotografie und Film am Ende des 20. Jahrhunderts mit Blick auf die Digitalisierung beider Medien und die Zunahme digitaler bildgebender Verfahren einen vielleicht vorerst letzten Schub bekommen. Dies ist auch auf die theoretischen Gewährsleute der Fototheorie Roland Barthes und Walter Benjamin zurückzuführen, denen in der Rezeption durch Bellour, Krauss, Dubois und andere eine

47 Schröter, »Intermedialität«, S. 137.

48 Schröter zitiert Joachim Paech, der mit Blick auf die Intermedialität die Untrennbarkeit von Medium und Form nach Niklas Luhmann ins Gedächtnis ruft. Luhmanns These, dass Formen und Medien aus jeweils feste beziehungsweise lose gekoppelten Elementen bestehen, hat er selbst auf das Kunstwerk als »unterscheidbare Form« angewendet (vgl. Niklas Luhmann, »Weltkunst«, in: ders., Frederick D. Bunsen, Dirk Baecker, Unbeobachtbare Welt. Über Kunst und Architektur, Bielefeld 199o, S. 7-45, S. 16). 
wichtige Rolle zukommt. In zahlreichen Monografien, ${ }^{49}$ Sammelbänden $^{50}$ und unzähligen Einzelaufsätzen, die vor allem in den Zehnerjahren des 21. Jahrhunderts zum Zusammenspiel von Fotografie und Film als Dichotomie von Stillstand und Bewegung in der Kunst erschienen, dominieren einige wenige foto- und filmtheoretische Positionen, die, wie in der Diskussion um die Indexikalität und Zeitlichkeit der Medien, stets auf Neue als Fixpunkte der Diskussion herangezogen werden. ${ }^{51}$

So ist es denn auch nicht verwunderlich, dass George Baker, um eine Position unter vielen herauszugreifen, den aktuellen Status des Fotografischen bedauert ${ }^{52}$ und sich dabei auf die vormals paradigmatische, aber auch umstrittene Rolle bezieht, die Krauss dem fotografischen Prinzip von Index und Kopie noch 1977 als wegweisend für die Moderne zugesprochen hatte. ${ }^{53}$ Künstlerische Fotografie stehe heute zwischen anderen Künsten, häufig unter dem Einfluss des malerischen Piktoralismus oder kinematografischer Mise en scène, oder werde begleitet von Videoprojekten. Sein Ziel, die Fotografie als Objekt wieder aufzubauen (»reconstruct«), kann jedoch eher als rhetorische denn historisch fundierte Volte gesehen werden, um angesichts des cinematic turn in den zeitgenössischen Künsten der Fotografie zu neuer Aufmerksamkeit zu verhelfen. Er wendet dafür das strukturalistische Krauss'sche Schema eines erweiterten Feldes der Skulptur auf die Fotografie an. In ihrem Modell wurde die Skulptur, die in den 196oer Jahren zu einer »Art ontologische[r] Abwesenheit « geworden war, als reine Negativität und Summe dessen, was sie alles nicht mehr sein konnte oder sollte, mittels einer Gruppe gegensätzlicher Kategorien neu bestimmt. ${ }^{54}$ Wo sich bei der Skulptur die Begriffe Landschaft und Nicht-Architektur ebenso wie Nicht-Landschaft und Architektur entsprachen,

49 Garrett Stewart, Between Film and Screen. Modernism's Photo Synthesis, Chicago 1999; Laura Mulvey, Death $24 x$ a Second. Stillness and the Moving Image, London 2006; Eivind Røssaak, The Still/Moving Image. Cinema and the Arts, Saarbrücken 2010.

Siehe unter anderem Green, Lowry, Stillness and Time; David Campany (Hg.), The Cinematic, London/Cambridge, MA 2007; Beckman, Ma, Still Moving; Diekmann, Gerling, Freeze Frames; Guido, Lugon, Fixe/animé; Røssaak, Between Stillness and Motion.

$5^{1}$ Beachtenswert ist in dieser Hinsicht auch, dass die gegenwärtige Rezeption der Fototheorie fast ausschließlich männliche Protagonisten berücksichtigt und Theoretikerinnen wie Susan Sontag kaum rezipiert werden. Für diesen Hinweis danke ich Katja Müller-Helle.

52 George Baker, »Photography's Expanded Field«, in: Beckman, Ma, Still Moving, S. 175-188.

53 Gemeint ist hier die Umdeutung der Fotografie als theoretisches Objekt. Krauss sieht die Fotografie qua ihrer Indexikalität und Deutungsfreiheit (Botschaft »ohne Code«) in Anlehnung an Pierce und Barthes als ein allgemeines Modell für die Logik der Gegenwartskunst an, je nach historischer Einschätzung also der Kunst der Moderne oder der Postmoderne.

54 Rosalind E. Krauss, »Skulptur im erweiterten Feld«, in: dies., Die Originalität der Avantgarde und andere Mythen der Moderne, Amsterdam 200o, S. 331-346, S. 338 f. 
ergänzen sich in der Fotografie bei Baker »not-narrative« und »stasis« wie auch »narrative« und »not-stasis«. ${ }^{55}$ In der Folge benennt er drei Modi des zwischen dem Narrativen und dem Statischen changierenden fotografischen Bildes: »still film«, zum Beispiel die projected images von Coleman, »talking picture « wie bei Jeff Wall, den er mit seinen an Pastiche und Historienmalerei angelehnten Werken in Opposition zur modernistischen Fotografie sieht, und schließlich Cindy Shermans »film stills«, die Baker cinematic photograph nennt. Es soll hier nicht der Ort sein, den Sinn einer strukturalistischen Herangehensweise an aktuelle fotografische Tendenzen im Detail zu bewerten; dass sie eine Möglichkeit darstellt, neue Begrifflichkeiten zu finden, zwischen denen sich die Formen fotografischer oder kinematografischer Bilder situieren können, ist jedoch zu bemerken. Und als solche will Baker sein Schema auch verstanden wissen. Vor einem Rückfall in traditionelle, konservative Diskurse über Medienspezifik warnt er ausdrücklich. Vielmehr soll mit einem erweiterten Feld von Begrifflichkeiten ermöglicht werden, die Randbereiche, die Grenz- und Übergänge von Fotografie, Film und anderen Medien nicht innerhalb einer einfachen Dichotomie, die sich an singuläre Charakteristika der Medien bindet, auszuloten, sondern in der Benennung transmedialer Eigenschaften die Künstler/innen und Werke in das entsprechende Bezugsgeflecht von Narrativität und Stasis einzuordnen. Dass auch Baker versucht, neue Ausdrücke für die jeweiligen ästhetischen Erscheinungsformen zu finden, wie beispielsweise cinematic photograph, führt uns zum letzten hier anzusprechenden Punkt, der Begriffsverwirrung, die die Forschung angesichts der zu beschreibenden Bilder zuweilen erfasst.

»A certain discomfort can be observed in the terminological vagueness or ambiguity when hybrid images between photography and cinema, still and moving images, are discussed. ${ }^{56}$ Diese Beobachtung machen Hilde van Gelder und Alexander Streitberger angesichts der Fülle von Versuchen, die seit dem Beginn des 20. Jahrhunderts auftretenden Transformationen des fotografischen, filmischen, videografischen und digitalen Bildes in Worte zu fassen. Die Versuche, den in den Künsten entstehenden neuen inter-, transund multimedialen Bildformen auch sprachlich beizukommen, haben sich oft als zu schwammig und ungenau herausgestellt.

Als Erstes etablierte Bellour den Ausdruck des entre-images, >zwischen den Bildern $<$, um darauf aufmerksam zu machen, dass die mannigfaltigen Seinsweisen des Bildes den Blick von einer ontologischen Fragestellung weg und hin

55 Baker, »Photography's Expanded Field «, S. 184.

$5^{6}$ Hilde van Gelder, Alexander Streitberger, »Photofilmic images in contemporary visual culture «, in: Philosophy of Photography 1/1, 2010, S. 48-53, S. 50. 
zu den Übergängen des Bildes beziehungsweise in das Zwischen den Bildern lenken müssten. ${ }^{57}$ Ausgehend vom Video als dem Medium, das Passagen eröffne, indem es in intermedialen Kunstformen die Intervalle und Räume zwischen den Bildern betone (zwischen Stillstand und Bewegung, zwischen der Fotografie und ihren Transformationen), ${ }^{58}$ charakterisiert er das entreimages als virtuellen Ort dieser Übergänge. Bellours Definition bleibt aufgrund seiner poetischen Sprache diffus und schwer zu übersetzen, weshalb es sinnvoll erscheint, hier eine längere Passage zur Charakterisierung des entreimages zu zitieren:

L'entre-images est ainsi (virtuellement) l'espace de tous ces passages. Un lieu, physique et mental, multiple. À la fois très visible et secrètement immergé dans les œuvres remodelant notre corps intérieur pour lui prescrire de nouvelles positions, il opère entre les images, au sens très général et toujours singulier $\mathrm{du}$ terme. Flottant entre deux photogrammes comme entre deux écrans, entre deux épaisseurs de matière comme entre deux vitesses, il est peu assignable: il est la variation et la dispersion même. C'est ainsi que les images désormais nous parviennent, l'espace dans lequel il faut décider quelles sont les vraies images. C'est-à-dire une réalité du monde, aussi virtuelle et abstraite soit-elle, une réalité d'image comme monde possible. ${ }^{59}$

Das entre-images stellt also keine Definition eines Bildes dar, sondern betont seine Latenz, es beschreibt den ephemeren Charakter von bildlichen Phänomenen im Übergang zwischen stabilen, benennbaren Zuständen. Korrespondierend zeigt sich Bellours Begrifflichkeit eben auch nicht historisch hergeleitet oder theoretisch fundiert, sondern sie basiert auf der Erfahrung sich ständig transformierender Bilder, die er als Eintritt in eine neue Zeit des Bildes wertet. ${ }^{60}$

Victor Burgin wählt den Zugang zum Film nicht über den Zwischenraum zwischen den Bildern, sondern über die Erinnerung: Um auszudrücken, dass er sich an seine erste filmische Erfahrung nicht über Einzelbilder, den Plot, die Schauspieler oder Details der Narration erinnert, verwendet er den Ausdruck sequence-image ${ }^{61} \mathrm{Im}$ Gegensatz zu image sequence, also einer fortlaufenden Reihung einzelner Bilder oder Einstellungen, ist dieses Sequenzbild stärker

57 Bellour, »Die Doppel-Helix«, S. 80, im Original Raymond Bellour, »La double hélice«, in: Passages de l'image, Ausstellungskatalog Musée national d'art moderne, Centre Georges Pompidou, Paris, Fundació Caixa de Pensions, Barcelona, Power Plant, Toronto, Wexner Art Center, Columbus, Modern Art Museum, San Francisco 199o-1992, Paris 199o, S. 37-56.

$5^{8}$ Bellour, »L'Entre-Images«, S. 14.

59 Ebd.

60 Ebd., S. 15 .

61 Victor Burgin, The Remembered Film, London 2004. 
als Objekt denn als Narration zu begreifen, eine willkürliche, sporadische Sammlung filmischer Eindrücke, die sich in der Erinnerung zu einem einzelnen Bild zusammenfügen, das in seiner Kürze jedoch weder als Sequenz noch als Einzelbild zu bezeichnen ist, sondern als »sequence of such brevity that I might almost be describing a still image ${ }^{62}$ Burgin beschreibt es - und dies ist interessant für die Fragestellung nach der terminologischen Vereinbarkeit der Zeitlichkeit und der Geschichtlichkeit des Bildes - als ein transitorisches, trotz allem jedoch faktisches Zusammentreffen eines aktuellen Moments der Wahrnehmung mit vergangenen Affekten und Bedeutungen:

[T] he sequence image as such is neither daydream nor delusion. It is a fact - a transitory state of percepts of a >present moment seized in their association with past affects and meanings, Pierre Nora has spoken of the sincreasingly rapid slippage of the present into a historical past that is gone for good ${ }^{63}$

Eine Zuordnung dieses komplexen Erinnerungsbildes zu einem medienspezifischen Diskurs erscheint aufgrund seiner assoziativen, poetischen Natur, die sich sprachlicher Logik widersetzt, unmöglich: »As this object - the sequenceimage - is neither image nor image sequence, it belongs neither to film nor to photography theory as currently defined. Indeed it may be doubted whether it can ever be fully a theoretical object, at least so long as theory remains an affair of language. «64 Burgin beschreibt mit dem sequence-image eine flüchtige Konstellation von Bildern, wie sie in der zeitgenössischen Film- und Videokunst häufig zu finden ist, und schafft damit trotz aller Vagheit einen Begriff, um sich diesen Bildern zu nähern. Man denke an Werke von Eija-Liisa Ahtila, Pierre Huyghe, Tacita Dean oder Hannes Schüpbach, in denen atmosphärische Bilder sich aufgrund ihrer Kürze jeder Beschreibbarkeit entziehen, jedoch wiederum zu lang sind, um mit Fotografien assoziiert zu werden. Hier spielt auch der von Burgin vernachlässigte Ton eine große Rolle.

Durch die Nutzung von Video und digitalen Methoden, um bewegte und unbewegte Bilder zu erzeugen, zu bearbeiten und miteinander zu kombinieren, haben sich die Unterschiede aufgeweicht. Bellour führt Video 1991 als das »letztgeborene unter den Reproduktionsbildern« auf, »ein Bild, das fähig ist, sämtliche vorausgegangenen Bilder - Malerei, Photographie, Film - anzuziehen, aufzulösen und miteinander zu vermischen ${ }^{65}$ Es habe jedoch noch

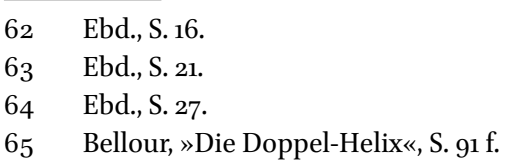


eine analoge Verbindung zur Welt, die ihm schließlich mit der digitalen Weiterverarbeitung ausgetrieben werde. Das Video als das nicht nur die Kunst, sondern auch den Alltagsgebrauch dominierende Bildmedium ab den 197oer Jahren wird von Douglas Gordon nicht umsonst für seine paradigmatischen Found-Footage-Arbeiten mit verlangsamten Spielfilmen verwendet: Es ermöglicht mittels seiner Intermedialität die Annäherung des ehemals kinematografischen Bildes an den Status eines Standbilds, ohne jedoch beides zu sein. ${ }^{66}$

So ist abschließend zu fragen, wie die dichotome Gegenüberstellung von Film und Fotografie in der Theorie, im Diskurs und in den Kunstwerken selbst historisch zu verorten ist. Dazu empfiehlt es sich, noch einmal einen Blick in die Filmgeschichte zu werfen, da im Experimentalfilm parallel zum Aufkommen der Videokunst die Spezifik des filmischen Mediums und seiner Rezeptionsbedingungen rekapituliert wurde. Der strukturelle Film stand in den 196oer und frühen 1970er Jahren für eine Bewegung von Künstler/ innen und Filmemacher/innen, die sich der Reduzierung des Films auf seine Materialität und die Grundelemente der filmischen Darstellung verschrieben hatten. Hollis Frampton, dessen Film (nostalgia) am Beginn dieses Kapitels stand, war ein Vertreter dieser Strömung, die vor allem in den USA, Großbritannien, Kanada und Österreich verbreitet war. Ihre Hauptvertreter/innen arbeiteten relativ getrennt voneinander, verfolgten jedoch ähnliche Ansätze. Sie versuchten, den Film mithilfe seiner eigenen Mittel zu definieren und auf die Grundbedingungen seiner Darstellung zurückzuführen, das heißt auf das Zusammenwirken von Kamera- und Projektionstechnik, Zelluloid, Raum und Zeit. Charakteristika waren die Sichtbarmachung von Effekten der Filmtechnik wie zum Beispiel das Flickern, der Freeze Frame und die Thematisierung der räumlichen Darstellung und dispositiven Anordnung von Apparat und Zuschauer/innen. ${ }^{67}$ Zwar wird der strukturelle Film stets unter

66 Video ist einerseits intermedial, da es auf elektronischer Grundlage vermag, die optischen Medien zu vermischen, es wurde aber selbst auch Gegenstand medienontologischer Diskussionen über seine Spezifität. Für die Videokunst der 196oer und 197oer typisch und bis heute maßgeblich ist Rosalind E. Krauss' These, das Medium des Videos sei der Narzissmus, es liege ihm also ein psychisches und kein physisches Modell zugrunde. Charakteristika seien vor allem die Simultaneität von Aufnahme und Projektion sowie die körperliche Rückkopplung zwischen Kamera und Monitor. Vgl. Rosalind E. Krauss, »Video. The Aesthetics of Narcissism«, in: October 1, Frühjahr 1976, S. 50-64, S. 52.

67 Zum strukturellen Film siehe einführend P. Adams Sitney, »Structural Film«, in: Film Culture 47, Sommer 1969, nicht paginiert; Peter Gidal, Materialist Film, London / New York 1989; Stephen Heath, »Repetition Time. Notes Around >Structural/materialist Film «", in: ders., Questions of Cinema, London/Basingstoke 1981, S. 165-175; Birgit Hein, Wulf Herzogenrath (Hg.), Film als Film - 1910 bis heute. Vom Animationsfilm der zwanziger zum Filmenvironment der siebziger Jahre, Ausstellungskatalog Kölnischer Kunstverein, Köln, Akademie der Künste, Berlin, Museum Folkwang, Essen, Württembergischer 
dem Diktum der Reinheit des Mediums genannt, das unter dem deutlichen Einfluss der modernistischen Kunsttheorie nach Clement Greenberg steht. ${ }^{68}$ In der Praxis wurde diese vor allem von P. Adams Sitney propagierte formelle Reinheit jedoch nicht konsequent verfolgt, wie Gabriele Jutz unterstreicht. Am Beispiel von George Landows Film in Which There Appear Sprocket Holes, Edge Lettering, Dirt Particles, Etc. (1965-1966) zeigt sie auf, dass die »Rhetorik des Puren« eine "zweifellos strategische und zeitgebundene Deutung des Avantgardefilms « darstellte. ${ }^{69}$

Eine andere Bewegung stellte zeitgleich das Expanded Cinema dar, eine Entwicklung hin zu Mehrfachprojektionen im Ausstellungsraum, zur Ausweitung des Kinos und Hybridisierung des Films durch Performance und Theater. Beide Bewegungen sind historische Vorläufer der zeitgenössischen Foto-, Film- und Videokunst, denn in ihnen wurden die Medien auf ihre spezifischen Eigenschaften und ästhetischen Möglichkeiten hin untersucht. Gemeinsam ist den Vertretern etwa ab den 199oer Jahren und ihren Vorgängern in der Filmgeschichte der reflektierende Umgang mit ihren Medien. ${ }^{70}$ Es geht ihnen nicht, zumindest nicht vornehmlich, darum, Geschichten zu erzählen, sondern zu ergründen, wie filmische Bildherstellung, Darstellung und Wahrnehmung funktionieren und welche psychischen Effekte eine Rolle spielen.

Die Gegenüberstellung von Frampton und Claerbout zeigte einige Gemeinsamkeiten, aber auch maßgebliche Unterschiede. Gemeinsam ist den Arbeiten und der Arbeitsweise der Künstler, dass sich abgesehen vom zeitlichen Verlauf der semantische Gehalt der Arbeiten mit Mitteln der Zeitlichkeit und ihrer Manipulation realisiert. Bei Frampton werden Vergangenheit und Gegenwart als Merkmale von Fotografie und Film und gesprochener Sprache miteinander vermischt; die zeitliche Verschiebung bewirkt auch eine Verschiebung des Bildobjekts, die statische Kameraeinstellung steht dem ständigen Austausch

Kunstverein, Stuttgart 1977/1978, Stuttgart 1977; Matthias Michalka (Hg.), X-Screen. Filmische Installationen und Aktionen der Sechziger- und Siebzigerjahre, Ausstellungskatalog Museum Moderner Kunst Stiftung Ludwig, Wien, 2004, Köln 2004.

68 Siehe hierzu vor allem den Essay »Modernist Painting« von 1960, erschienen in Forum Lectures, Washington D. C., auf Deutsch: Clement Greenberg, »Modernistische Malerei«, in: ders., Die Essenz der Moderne. Ausgewählte Essays und Kritiken, hg. v. Karlheinz Lüdeking, Dresden 1997, S. 265-278.

69 Gabriele Jutz, Cinéma brut. Eine alternative Genealogie der Filmavantgarde, Wien / New York 2010, S. 35 .

70 Vgl. hierzu u. a. Carla Taban (Hg.), Meta- and Inter-Images in Contemporary Visual Art and Culture, Leuven 2013. Taban sieht einen Zusammenhang zwischen der Verbreitung digitaler Medien in der Kunst, der systematischen theoretischen Erörterung »metavisueller« Phänomene und der kritischen, interdisziplinären Diskussion eines »pictorial/ iconic/visual turn«. Vgl. dies., »Introduction«, ebd., S. 11-40, S. 27. 


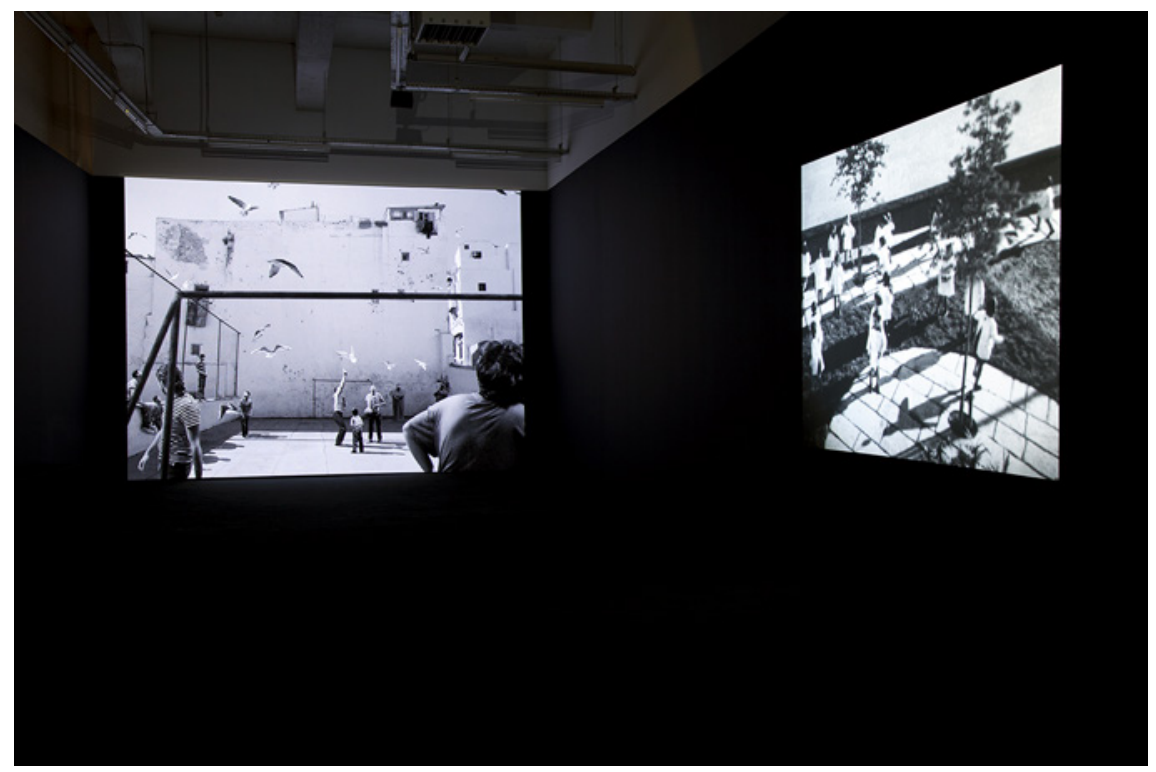

Abb. 6

David Claerbout, The Algiers' Sections of a Happy Moment, 2008, Ausstellungsansicht Wiels, Brüssel, 2011

und Verschwinden der Fotografie entgegen. Bei Claerbout treffen im digitalen Video Fotografie und Bewegung aufeinander. Das in seiner starren Künstlichkeit wie der Zeit entrissen wirkende Foto wird durch die unwirkliche Bewegung gestört. Claerbout macht sich, auch in anderen Werken, die latente Bewegung des Videobildes zunutze, um Standbilder nur minimal zu verändern und dadurch einen Eindruck von Langsamkeit und Dauer zu erzeugen, der ähnlich wie bei Gordon eine erhöhte Aufmerksamkeit für das Bild einfordert.

Der Umgang mit Film und Fotografie ist denn auch sehr unterschiedlich. Für Frampton gehören sie aufgrund ihres optisch-mechanischen Ursprungs der gleichen Sphäre an und gehen im Film somit auch eine inhaltliche Verbindung ein, verkettet durch die Erzählung des Voiceovers. Die zu seiner Zeit radikal neue Videotechnik als Symptom des elektronischen Zeitalters bildet die historische Schwelle, an der Film und Fotografie für Frampton obsolet werden und als historische Phänomene zur Kunst werden können (ähnlich argumentiert auch Rosalind Krauss, worauf im nächsten Abschnitt eingegangen wird). Claerbout, der mit Video und digitaler Projektion arbeitet, generiert in seinen Werken mittels der Bewegung und Stillstellung Sinnbilder für Fotografie und Film, die auf deren mediale Eigenschaften verweisen, diese jedoch nicht (mehr) zu definieren suchen. So kommen beispielsweise auch in der Arbeit The Algiers' Sections of a Happy Moment (2008) (Abb. 6), in der aus 
tausenden Fotos 6oo Bilder digital zusammengesetzt und zu einer 37 Minuten langen digitalen Projektion gemacht wurden, weniger die ontologischen als die ästhetischen Merkmale von Fotografie und Film zum Tragen. Claerbout agiert nicht als Historiker, der die Bilder zu einer alternativen Geschichtsschreibung nutzt, sondern entwirft durch die Manipulation der Zeitlichkeit der Fotografie mittels der Bewegung, die die Erfahrung des Bildes an die gegenwärtige Aufmerksamkeit der Betrachter/innen bindet, ein Bild, das die verschiedenen zeitlichen Dimensionen von Fotografie und Film zur Komposition von etwas Neuem nutzt.

\section{Post-Medium Condition. >Erfindung $<$ eines neuen Mediums?}

Die Diskussion über Intermedialität und Medienspezifik, speziell über den zeitgenössischen Gebrauch von Fotografie im Kontext kinematografischer Installationen, greift Rosalind E. Krauss 1999 erneut auf und aktualisiert dabei die modernistische Thematik der Reinheit und Autonomie des Mediums. ${ }^{71}$ Sie bezieht sich kritisch auf ihren Mentor Greenberg und dessen Doktrin der »flatness « als wichtigster Eigenschaft moderner Malerei, die in der Flachheit des Trägermediums, also der Materialität der Leinwand, fundiert ist. Dennoch möchte sie den Begriff des Mediums aufgreifen, jedoch ungleich weiter fassen:

Der postmedialen Verfasstheit des Mediums, wie sie Krauss beschreibt, eignet eine Spezifik, die sie nicht an den im modernistischen Diskurs vorbelasteten Begriff des Materials knüpft, sondern zunächst einmal vage als >differenziell beschreibt. Über verschiedene historische Positionen benennt sie Merkmale dieses Mediums: seine >innere Pluralität $<$ (Cavell), eine >aggregierende < Verfasstheit (struktureller Film) und >rekursive Struktur< (Denis). ${ }^{72}$

Krauss argumentiert in zwei Richtungen: einerseits beinhalte die genannte Selbstdifferenz des von ihr als postmedial bezeichneten Mediums eine Schichtung von Konventionen und sei niemals mit der reinen Materialität des Trägers in eins zu setzten, andererseits stelle die Erfindung bzw. Wiedererfindung eines solchen $>$ neuen $<$ Mediums immer eine historische Entwicklung

71 Krauss, $A$ Voyage on the North Sea.

72 Steinmüller, » To ferry images of presence and absence ««, S. 125. Dass das Konzept eines selbstdifferenziellen Mediums auch auf das Dispositiv der Bildprojektion anzuwenden und somit auf andere Künstler/innen zu übertragen ist, ist die Hauptthese dieses Aufsatzes. Die im Folgenden skizzierte Idee, die Projektion als Medium im Sinne Krauss' zu begreifen, geht auf die Ausführungen in diesem Aufsatz zurück. 
dar, indem Techniken veralteten und aus einem anderen Kontext in die künstlerische Nutzung überführt würden:

First, that the specificity of mediums, even modernist ones, must be understood as differential, self-differing, and thus as a layering of conventions never simply collapsed into the physicality of their support. [...] Second, that it is precisely the onset of higher orders of technology - >robot, computer - which allows us, by rendering older techniques outmoded, to grasp the inner complexity of the mediums those techniques support. ${ }^{73}$

Dass das Medium somit als Verbund einer offenen Trägerstruktur und ihres künstlerischen Gebrauchs zu verstehen ist, führte Juliane Rebentisch zu der berechtigten Kritik an der Unklarheit des Medienbegriffs dieser Stelle: Betrachte man Krauss' Beschreibungen im Lichte der Unterscheidung von Medium und Form nach Luhmann, ${ }^{74}$ also der Intensität der Koppelung von Elementen, welche die bestimmbare Form beeinflusst, so könne ihre Rede von der »Selbstdifferenz des ästhetischen Mediums« präzisiert werden.

Die Idee von Krauss, bestimmte Tendenzen der aktuellen Kunst in einem postmedialen Zeitalter zu verorten, schließt an ihre frühere, umstrittene, nichtsdestotrotz jedoch einflussreiche These an, dass die Fotografie als theoretisches Objekt beziehungsweise Prinzip der Gegenwartskunst gelten könne. Hierbei handelte es sich nicht um eine Theorie der Fotografie im eigentlichen Sinn, die eine Beschreibung der Spezifika des technischen Mediums, eines Kunstwerks oder einer Kunstform lieferte, vielmehr thematisierte Krauss ausgehend von der Fotografie die Übertragung eines funktionalen Prinzips von einem Gegenstand auf einen anderen. Beruhend auf ihren zwei viel beachteten Schriften zum Index hieß dies, »das Fotografische « - definiert einerseits als das Prinzip des Indexes nach Pierce, andererseits als »Botschaft ohne Code « nach Barthes - als Modell für die Logik der sogenannten postfotografischen Kunst zu begreifen. ${ }^{75}$ Anders beim nun propagierten Postmedialen, das sich als Ver-

73 Krauss, $A$ Voyage on the North Sea, S. 53.

74 Vgl. Niklas Luhmann, Die Kunst der Gesellschaft, Frankfurt am Main 1995, S. 165 ff., zit. n. Juliane Rebentisch, Ästhetik der Installation, Frankfurt am Main 2003, S. $84 \mathrm{f}$.

75 Rosalind E. Krauss, »Notes on the Index. Seventies Art in America«, in: October 3, Frühjahr 1977, S. 68-81, und dies., »Notes on the Index. Seventies Art in Amerika. Part 2«, in: October 4, Herbst 1977, S. 58-67. Vgl. auch dies., Das Photographische. Eine Theorie der Abstände, München 1998. Kritik an Krauss' Verknüpfung des Indexikalischen mit der Bedeutungsstruktur des fotografischen Bildes sowie die enge Knüpfung an eine Fortschrittsgeschichte der Gegenwartskunst findet sich u. a. bei Beat Wyss, »Das Fotografische und die Grenzen des mechanischen Bildes«, in: Hans Belting, Dietmar Kamper, Ingo Schulz (Hg.), Quel corps? Eine Frage der Repräsentation, München Verlag 2002, S. 365-376, S. 369, sowie zuletzt bei Geimer, Theorien der Fotografie zur Einführung, S. 25-31. 
such äußert, von der Medienspezifik ihres Lehrers Greenberg wegzukommen und ein »aggregierendes « Medium zu benennen, das genau den Übergang zwischen konkret zu fassenden technischen und medialen Erscheinungsformen und dem Umgang mit diesen markiert. Dass sie weiterhin als Kunsthistorikerin spricht, sieht man an ihrem Bestreben, diesen Prozess historisch zu verorten: Es sind vor allem die ausgedienten, anachronistischen Medien, die im neuen Gebrauch eine veränderte Bestimmung finden und in dieser ästhetischen Überformung ein gänzlich neues Medium bilden. ${ }^{76}$ Sie lassen sich als personalisierte, künstlerische Handschriften benennen wie bei ihren Beispielen Wall oder Coleman. Colemans Diainstallationen dienen Krauss als Ausgangspunkt der »Wiedererfindung « eines Mediums. Was für die Fragestellung dieser Untersuchung nach der Korrelation von Zeitlichkeit und Geschichtlichkeit in den Werken der Gegenwartskunst von besonderem Interesse ist, ist die Verknüpfung einer medialen Eigenheit mit ihrer unhintergehbaren Historizität, denn Krauss bezieht sich in fast allen ihren Beispielen (Wall, Coleman, Kentridge, Marker, Snow, Broodthaers) auf die gewandelte Verwendung von Fotografie und Film: James Coleman hat in seinen zahlreichen Diaarbeiten seit den 1970er Jahren eine typische, wiedererkennbare Form der Kombination komplexer fotografischer Bilderserien, die mittels synchronisierter Projektoren überblendet werden, und eines Voiceovers geschaffen und die Diaprojektion damit aus dem Abseits des heimischen Gebrauchs, der akademischen Nutzung oder der Verwendung in der Werbung herausgelöst; Jeff Wall hat den fotografischen Leuchtkasten, der in Zeiten vor großformatigen LED-Bildschirmen die Außenwerbung dominierte, zur Präsentation seiner Dias entdeckt; William Kentridge verknüpft Kohle- und Kreidezeichung mit filmischer Animation und raumgreifender Projektion; Chris Markers legendärer Fotofilm La Jetée (1962) verbindet das fotografische Bild mit dem Film entlehnter narrativer Dramaturgie und Montagetechnik sowie mit Konventionen wie dem Close-up, Flashbacks und Schuss-Gegenschuss-Einstellungen und erfindet so ein neues Kino. ${ }^{77}$

${ }_{7} 6$ Rebentisch merkt an, dass Krauss weniger auf die Erfindung neuer Medien abziele denn auf die Erschließung neuer künstlerischer Gattungen. Die Übertragung eines Mediums in einen ästhetischen Bereich zeuge zwar von einem reflektierten Umgang damit, schaffe aber noch keine eigene »ästhetische Autonomie« des Künstlers oder Werks. Vgl. Rebentisch, Ästhetik der Installation, Anm. 19, S. 99. Dieser Kritik stimme ich zu, Krauss' Medienbegriff bleibt unklar.

77 »La Jetée (1962) [...] seems to break with the cinematic medium, perhaps to found a new one«. (Rosalind E. Krauss, » ... And Then Turn Away?«. An Essay on James Coleman«, in: October 81, Sommer 1997, S. 5-33, S. 7.) 
Krauss bezieht sich dabei einerseits auf die bereits beschriebene Selbstdifferenz des verwendeten supports, in Colemans Fall auf das Dispositiv der Diainstallation, welches den fotografischen Bildträger, seine Projektion und die für Coleman spezifischen narrativen, an filmische und populärliterarische Erzählstrukturen angelehnte Bilderfolgen einschließt. Das projizierte Bild hat immer eine zeitliche und eine räumliche Dimension, es setzt eine apparative Struktur und eine/n sich dazu positionierende/n Betrachter/in voraus - insbesondere bei Coleman, der jegliche Aufzeichnung seiner Arbeiten untersagt und so die Erfahrung seiner Werke im Ausstellungsraum festlegt. Andererseits ist das >erfundene< Medium der Diainstallation (oder des Fotofilms bei Chris Marker, des Schaukastens bei Jeff Wall etc.) bei Krauss kein theoretisches, sondern ein historisches Objekt, da die traditionelle 〉Codierung < des jeweiligen Trägers, seine >Grammatik« oder `Syntax «, in dieses eingeschrieben ist und die Basis der aktuellen künstlerischen Umdeutung bildet. Der zweite Aspekt, dass es der Veralterung der jeweiligen Technik bedürfe, um sie in einen neuen, künstlerischen Kontext überführen zu können, in dem erst ihr imaginatives Potenzial zur Geltung komme, ergibt in Bezug auf die projected images Sinn, wird hier doch die gesamte Sphäre der Projektionskünste, von der Laterna magica bis hin zur Kinematografie, aufgerufen:

For Coleman as well, this resource of the magic lantern show, lodged within the commercial slide tape as a kind of genetic marker, is central to his project. It tells of an imaginative capacity stored within this technical support and made suddenly retrievable at the moment when the armoring of technology breaks down under the force of its own obsolescence. To >reinvent the slide tape as a medium - as I am claiming it is his ambition to do here - is to release this cognitive capacity, thereby discovering the redemptive possibilities within the technological support itself. ${ }^{78}$

Warum die Veralterung in einer allgemeinen Theorie des Mediums im spostmedialen Zeitalter $<$ jedoch zur zwangsweisen Voraussetzung gemacht werden müsse, erklärt Krauss nicht, und diese Einschränkung ist auch mit Blick auf die aktuellen Künste nicht überzeugend, wie ich bereits an anderer Stelle argumentiert habe:

Mit der Voraussetzung, dass erst die >obsolescence $<$ die notwendige Offenheit eines Mediums ermöglicht, hängt Krauss jedoch einem technikzentrierten Anachronismus nach, der ihre Medienkonzeption einschränkt und ihre Argumente schwächt. Sie kann weder den neuen, auf Video oder Computer basierenden

78 Rosalind E. Krauss, »Reinventing the Medium«, in: Critical Inquiry 25/2, Winter 1999, S. $289-305$, S. 304. 
Bildern, noch der Installations- und Multimediakunst etwas abgewinnen, obgleich sich die Idee einer medienimmanenten Differenz, die jeder ontologischen Bestimmung eines Mediums vorgängig ist, grundsätzlich auch auf neuere Techniken und Praktiken übertragen ließe. ${ }^{79}$

Krauss' Abneigung gegen intermediale Phänomene ist schon deshalb nicht nachvollziehbar, weil doch genau dies bei Coleman passiert: ein Zusammentreffen von Fotografie, Film und anderen Darstellungsformen wie dem Theater oder der Malerei, die sich jedoch nicht zu einem Undeutbaren vermischen, sondern an den jeweiligen Rändern >aneinanderstoßen<. Die in dieser Arbeit besprochenen Werke James Colemans machen anschaulich, dass im Medium der Diaprojektion die kinematografische Installation, die narrative Montage und die fotografische Tradition des Posierens miteinander kombiniert werden können, ohne dass die einzelnen Komponenten miteinander verschmelzen. Sprachliche und bildliche Ausdrucksformen berühren sich nur, sind aber auch nicht voneinander $\mathrm{zu}$ trennen, wie Baker in seiner Rede von einem »konsequenten >Teilen« von Formen« treffend bemerkt:

An interstice between mediums has not been >crossed<; forms can only share themselves around that which they lack. Forms can only (truly) share themselves around an absolute limit, a limit that must be respected, and yet this limitation is a gift. For this limitation also means that forms have an outside through which they can - or perhaps even must become other. ${ }^{80}$

Dass in der Diaprojektion nicht nur das Intervall zwischen den einzelnen Fotografien thematisiert, sondern auch der Zwischenraum zwischen den Gattungen und ihren bildlichen Traditionen reflektiert wird, birgt das Potenzial, die Bewegung und Beweglichkeit der Bilder mit der Historizität ihrer Darstellungsformen parallel zu betrachten.

Diese und andere hybride Werkformen, die sich vor allem seit den 199oer Jahren in den Ausstellungsräumen finden, fordern die mediale Einordnung einerseits, die phänomenologische Beschreibung der bewegten Bilder

79 Steinmüller, »»To ferry images of presence and absence««, S. 127. Vgl. auch die Kritik von Mary Ann Doane an Krauss: »This is a restricted specificity that takes the individual work and its activation of particular conventions as its point of departure, and not the medium itself. Those works that can be labeled `medium specific $<$ are those reiterating and reconfirming the constraints of their material support. Yet, it is a strange type of specificity that is selective (Mary Ann Doane, »The Indexical and the Concept of Medium Specificity«, in: differences 18/1, 2007, S. 128-152, S. 131).

8o George Baker, »Reanimations (I)«, in: James Coleman. Drei Filmarbeiten, Ausstellungskatalog Sprengel Museum Hannover, Hannover 2002, S. 15-8o (dt.) und S. 90-138 (engl.), S. 108 . 
andererseits heraus. Beides ist nicht voneinander zu trennen: Wo in projizierten Fotografien, Filmen oder Videos die Zeit als gestaltendes Material eingesetzt wird und eine strikte Einteilung in statische Bilder und filmische, bewegte Bilder keinen Sinn mehr zu ergeben scheint, da scheitern auch Versuche, die Werke mittels medienspezifischer Kategorien zu beschreiben, wie im ersten Teil des Kapitels hergeleitet wurde. Die zeitliche Nähe einer theoretischen Diskussion um die Spezifika der Medien Fotografie und Film, die in ihrer analogen Technik, Handhabung und Ästhetik angesichts von Video und Digitalität zunehmend anachronistisch wurden, zu den hybriden Bildformen in der Kunst lässt eine gegenseitige Befruchtung annehmen, auch weil manche Akteure wie Raymond Bellour als Kuratoren und Kritiker gleichermaßen in Erscheinung traten. Die Zeitlichkeit des fotografisch basierten Bildes schreibt sich, so ist schließlich festzustellen, zwischen die Extreme von Stillstand und Bewegung, in das Intervall des entre-images ein. So, wie sich die Zeitlichkeit des Bildes in der Korrelation von Bewegungsmotiven und ihrer Wahrnehmung zeigt, in der Zeit des Dargestellten wie auch der Darstellung (vgl. Kapitel 1), so lässt sich der Eindruck von Stasis und Dauer auf der einen Seite, von Bewegung auf der anderen Seite eben nicht nur an der materiellen, semantischen, semiotischen oder phänomenologischen Ebene des Werks festmachen, will man der Komplexität von Fotografie und Film Rechnung tragen. Es ist vielmehr der Bereich zwischen den Bildern, wo sich die Formen treffen, gegenseitig affizieren und überlagern. Ästhetische Strategien wie die Verlangsamung tragen dazu bei, von einer medienspezifischen Ontologisierung abzurücken und das Verstreichen der Zeit als Charakteristikum des bewegten Bildes stärker wahrzunehmen. 